\title{
Characterization of the Photochemical Properties of 5-Benzyluracil via Time-Dependent Density Functional Theory
}

\author{
M. Micciarelli, ${ }^{\dagger}$ B. F. E. Curchod, ${ }^{\ddagger}$ S. Bonella, ${ }^{\S}$ C. Altucci, ${ }^{\|}$M. Valadan, ${ }^{\|}$U. Rothlisberger, ${ }^{\ddagger}$ \\ and I. Tavernelli*, $\perp^{+}$ \\ ${ }^{\dagger}$ Department of Physics, University of Rome "La Sapienza”, Ple A. Moro 5, 00185 Rome, Italy \\ ${ }^{\ddagger}$ Laboratory of Computational Chemistry and Biochemistry and ${ }^{\S}$ CECAM Centre Européen de Calcul Atomique et Moléculaire, \\ École Polytechnique Fédérale de Lausanne, CH-1015 Lausanne, Switzerland \\ "Department of Physics, University of Naples "Federico II", Via Cintia, 26-80126 Napoli, Italy \\ ${ }^{\perp}$ Zurich Research Laboratory, IBM Research GmbH, 8803 Rüschlikon, Switzerland
}

ABSTRACT: We present a detailed study of the excited state properties of 5-benzyluracil $(5 B U)$ in the gas phase and in implicit solvent using different electronic structure approaches ranging from time-dependent density functional theory in the linear response regime (LR-TDDFT) to a set of different wavefunction-based methods for excited states, namely perturbed coupled cluster (CC2), algebraic diagrammatic construction method to second order $(\mathrm{ADC}(2))$, and perturbed configuration interaction (CIS(D)). 5BU has been used to investigate DNA base-amino acid interactions. In particular, it served as a model of protein-DNA photoinduced cross-linking. While LRTDDFT is computationally the most efficient first-principles approach for static and dynamic simulations of this bichromo-

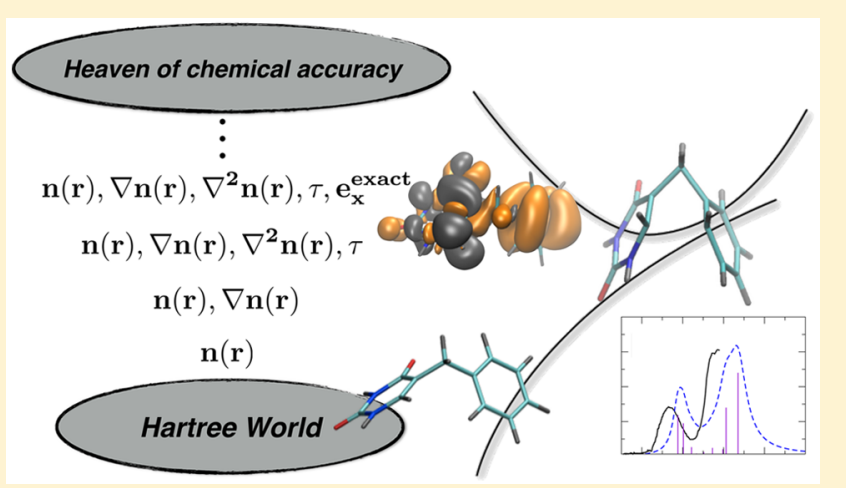
phoric system, its accuracy is difficult to assess due to the presence of excited states with charge transfer character. In this work, the performance of different exchange correlation functionals is compared against accurate benchmarks obtained either from high level wave-function-based methods or directly from experimental absorption spectra. Our investigation shows that accurate results for the excitation energies can be obtained using the hybrid meta-GGA functional M06. In view of dynamical studies of the relaxation of 5BU after photoexcitation, we also show that the PBE functional, while failing in the Franck-Condon region, provides qualitatively good results for the characterisation of a possible photocyclization path.

\section{INTRODUCTION}

5-Benzyluracil (5BU) is a RNA/DNA base derivate obtained from uracil (Figure 1a) by formally replacing a hydrogen atom with a phenyl group (via a methyl bridge) in position 5 or, equivalently, from thymine (Figure $1 \mathrm{~b}$ ) adding phenyl directly to the methyl group. This molecule allows the study of the proximity interactions of a pyrimidine nucleobase with an aromatic chromophore (the uracil moiety playing the role of a DNA/RNA base pair). The two aromatic moieties can be interpreted, in biological terms, as representative components of DNA/RNA base pairs (the uracil ring) and aromatic protein amino acids (e.g., phenylalanine or tyrosine). The two rings are kept in a favorable configuration for cross-linking by the methyl linker (Figure 1). After UV irradiation one observes the formation of 5,6BU, a cyclized structure in which a covalent bond between the carbons in position 6 and 10 is formed (Figure 1c,d). This reaction can be used as a local model of photoinduced DNA protein cross-linking. ${ }^{1}$ Because of their biological relevance, the photophysics and photochemistry of $5 B U$ have been recently studied combining steady state spectroscopy and calculations based on linear response-time dependent density functional theory (LR-TDDFT). ${ }^{2}$ It has been shown that most of the principal properties characterizing the DNA pyrimidine nucleobases (excited states assignment, absorption and emission transitions, ultrafast electronic radiative decay time scale) are not perturbed by the chemical addition of the phenyl group in 5BU. However, it was also shown that in 5BU an excited state with strong charge transfer (CT) character is present, with partial hole on the benzene and excited electron on the uracil. This state is particularly important for the photoreactivity of the molecule since it appears to be the most likely channel for the photocyclization reaction.

The electronic structure calculations performed to obtain the results reported in ref 2 are particularly challenging due to the bichromophoric nature of this system. In fact, it is well documented that LR-TDDFT often underestimates excitation energies involving states with strong CT character. ${ }^{3-5}$ In

Received: December 20, 2016

Revised: $\quad$ May 2, 2017

Published: May 3, 2017 
<smiles>O=c1cc[nH]c(=O)[nH]1</smiles>

a) Uracil<smiles>O=c1[nH]cc(Cc2ccccc2)c(=O)[nH]1</smiles>

c) $5 \mathrm{BU}$

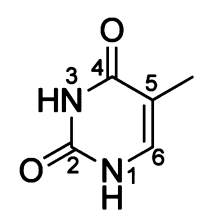

b) Thymine

Figure 1. Molecular Lewis structure of (a) uracil, (b) thymine, (c) 5benzyluracil (5BU), and (d) its photoproduct $(5,6 \mathrm{BU})$. The atoms in the cyclic and heterocyclic rings have been labeled to facilitate discussion in the text.

general, testing the accuracy of LR-TDDFT (or DFT) calculations is not trivial, since the results depend on the choice of the exchange correlation (xc) energy functional employed. Most functionals are constructed using physical principles and/or fitting procedures, and it is difficult to systematically assess their relative performances in the absence of reference data. On the other hand, the use of post-HartreeFock multideterminant methods to fully characterize the excited states of 5BU is not possible with reasonable computational cost. Indeed, it was recently shown in studies of 5BU in the gas phase at the CASSCF-CASPT2 level of theory ${ }^{6}$ that the size of the affordable active space does not allow an adequate description of the first excited states of this molecule in the Franck-Condon region. Alternatively, semiempirical approaches ${ }^{7,8}$ (such as PM3, OM2, and OM3) could be used, but these require parametrization and validation processes that go beyond the scope of this work.

In this work, we extend our analysis of LR-TDDFT for the calculation of the electronic structure of 5BU in the gas phase and in implicit solvent with the aim of assessing (using a systematic approach) the most accurate exchange and correlation (xc) functional for the description of the photoreaction mechanism. This is done using different standard approximations for the $\mathrm{xc}$ functional and comparing the results with benchmark calculations performed in gas phase and in implicit solvent models. When available, the LR-TDDFT results are compared to experimental spectra obtained in solution. We proceed following the conceptual ranking of the approximated xc functionals proposed in 2001 by Perdew and Schmidt, ${ }^{9}$ known as the "Jacob's ladder" for DFT/LR-TDDFT functionals, to which we will refer in the following as the Perdew's ladder. We test the performance of popular functionals belonging to different rungs of the ladder, and we compare their performance by considering in particular (i) the absolute and relative energies of the states, (ii) the assignment of the character of the different excited states, and (iii) the accuracy of the energies of the CT states. This last analysis is particularly relevant given its significance for our study on molecular photochemistry and the known failures associated with the calculation of CT excitation energies within LRTDDFT. ${ }^{3-5}$

Finally, we also investigate the possibility to use the generalized gradient approximation (GGA) of the xc energy functional for the description of the putative reactive decay path

connecting $5 \mathrm{BU}$ to the photoproduct 5,6BU along the $\mathrm{CT}$ potential energy surface (PES). As we will show in the following, even if in the Franck-Condon region the GGA functionals (we tested, in particular, the $\mathrm{PBE}^{10}$ ) describe very poorly the global manifold of excited states, the accuracy of the CT PES in regions of the molecular configurational space along the reactive decay path (and therefore away from the FranckCondon region) improves significantly. Although further validation may be required, the low computational cost associated with this approximation in plane waves based firstprinciples codes makes it an interesting candidate for studying the cyclization mechanism using on-the-fly nonadiabatic molecular dynamics techniques. ${ }^{11-14}$

\section{COMPUTATIONAL DETAILS}

The LR-TDDFT calculations were performed using six different popular xc functionals belonging to different rungs of the Perdew's ladder ${ }^{9,15}$ sketched in the scheme below for the reader's convenience.

$$
\begin{gathered}
F_{\text {double-hybr,RPA }}\left[n(r), x(r), \tau(r),\left\{\phi_{i}\right\},\left\{\phi_{a}\right\}\right] \\
F_{\text {meta-hybr }}\left[n(r), x(r), \tau(r),\left\{\phi_{i}\right\}\right] \\
F_{\text {hybr }}\left[n(r), x(r),\left\{\phi_{i}\right\}\right] \\
F_{\text {meta-GGA }}[n(r), x(r), \tau(r)] \\
F_{\mathrm{GGA}}[n(r), x(r)] \\
F_{\mathrm{LDA}}[n(r)]
\end{gathered}
$$

In this notation, $n(r)$ is the electronic density, $x(r)=|\nabla n(r)| /$ $n(r), \phi_{i}(r)$ the Kohn-Sham orbitals, $\tau(r)=\sum_{i} f_{i} \phi_{i}(r) \nabla^{2} \phi_{i}(r)$ ( $f_{i}$ is the occupation), and we use the index $i$ for occupied and $a$ for unoccupied orbitals. As discussed in the following, in the case of 5BU sufficient accuracy was obtained with meta-hybrid functionals, and therefore representatives of the last rung were not tested. In our calculations we chose $\mathrm{PBE}^{10}$ in the GGA, M06 $\mathrm{L}^{16}$ in the meta-GGA, B3LYP ${ }^{17}$ in the hybrid, and $\mathrm{M} 06^{18}$ in the meta-hybrid functional classes. Two more meta-hybrid functionals were also included: (i) the range separated CAM$\mathrm{B} \mathrm{LYP}^{19}$ functional to study possible long-range effects associated with CT transitions and (ii) the M06-2X ${ }^{18}$ functional (which has recently been shown to perform as well as or, in cases with intermediate spatial overlap, better than CAM$\left.\mathrm{B}_{3} \mathrm{LYP}^{20}\right)$. In all cases the Dunnings cc-pVTZ ${ }^{21}$ correlation consistent basis set was used. For the numerical integrations of the xc energy we used a grid, with 75 radial shells and 302 angular points per shell. As convergence criterium we used a threshold of $10^{-6}$ au for energy convergence and of $10^{-8}$ in the root-mean-square (RMS) deviation of the density matrix.

To compare the different performances, we computed the excited state energies and characters using for all functionals the same optimized molecular geometry obtained with the M06 functional. A description of this structure is given in Ref 6, where the most relevant geometrical parameters (bond lengths and Wiberg's bond indexes) are discussed. CIS(D) calculations were performed with Gaussian09, while Turbomole ${ }^{22}$ was used for the $\mathrm{CC}^{23}$ and $\operatorname{ADC}(2)^{24,25}$ calculations. All these calculations used a cc-pVTZ basis set. ${ }^{21}$

Calculations of 5BU solvated in $\mathrm{MeOH}$ were also performed to compare with experimental absorption data and to investigate the decay channel leading to the experimentally observed photocyclization. The solvent was represented 


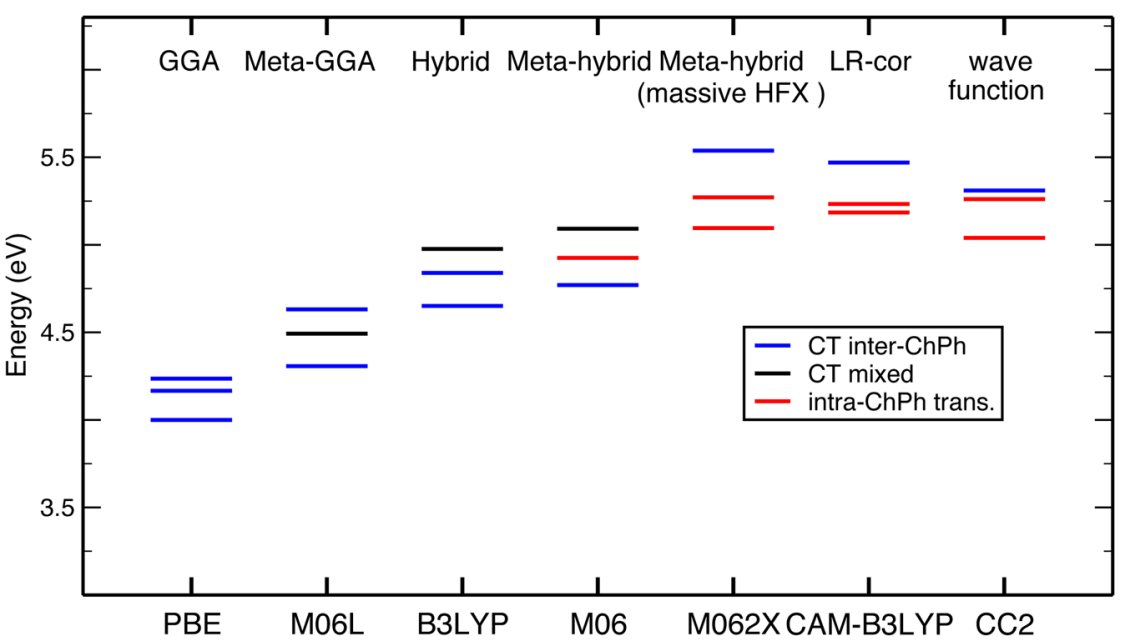

Figure 2. Vertical excitation energies to the first three excited states computed using different electronic structure methods. For all calculations the same optimized molecular geometry obtained with the M06 functional is used. Different standard xc functionals are arranged following their order on the Perdew's ladder. The last column reports results obtained using wave function based electronic structure methods (CC2). The different line colors describe different excitation characters. CT inter- $\mathrm{ChPh}$ (blue): interchromophore charge transfer transitions; CT mixed (black): charge transfer states with inter- and intrachromophore character; intra-ChPh trans (red): intrachromophore transitions. Intrachomophore transitions (within the uracil or benzene moiety) can have $\pi \pi^{*}$ as well as $\mathrm{n} \pi^{*}$ character.

implicitly and treated as a continuum dielectric medium using the polarizable continuum model (PCM) in its integral equation formalism (IEFPCM $)^{26}$ variant. In this representation, the solvent is characterized solely by its relative static dielectric permittivity $\epsilon_{\mathrm{r}}$ that we set to 32.61 . $^{27}$

To model the vertical absorption in the solvated phase, full geometry optimizations of $5 \mathrm{BU}$ in the electronic ground state were performed with different functionals. For each ground state optimized geometry, the electronic excitation energies were obtained and compared to experiments when available. To cover the experimentally observed energy range, the first 10 vertical excitations have been taken into account. Simple Lorentzian functions envelopes (with full width at halfmaximum of $0.25 \mathrm{eV}$ ) have been used to model the FranckCondon lines broadening and take into account the effect of thermal broadening.

It has been shown that the inclusion of thermal broadening in the calculation of absorption spectra can play an important role in the description of optical absorption of molecules. ${ }^{28-31}$ In particular, using first-principles $\mathrm{MD}$, it is possible to capture the asymmetric broadening of the different absorption peaks and the corresponding peak shifts. However, in this work we did not consider thermal effects since the static results were already sufficient to assess the performance of the different functionals.

To further analyze the results obtained for the excited states, we have also quantified the overlap of the orbitals participating to the CT excitations using the $\Lambda$ diagnostic parameter ${ }^{32}$ as implemented in the Gamess program suite. ${ }^{33}$ For these calculations we used the same basis set (cc-pVTZ) and xc functionals adopted in the other calculations performed with Gaussian09.

Within Gamess, ${ }^{33}$ we used a Lebedev grid with 96 radial points and 770 angular points, an energy threshold of $10^{-6} \mathrm{au}$ was used for the self-consistent field convergence, and a PCM approach with charge scaling set to $\epsilon_{\mathrm{r}}-1 / \epsilon_{\mathrm{r}}$ was applied to model the solvation in $\mathrm{MeOH}$. These changes in the simulation conditions did not cause significative variations in the results. In particular, no changes were observed in the energies, transitions assignment, and oscillator strengths. To better evaluate the influence of exact exchange on the results, the Gamess program suite was also used to tune (in the gas phase) the range separation parameter $\gamma$ in the long-range corrected version ${ }^{34}$ of the GGA functional BLYP ${ }^{35}$ (BLYP was chosen to compare directly with CAM-B3LYP). As suggested by Stein et al., ${ }^{36}$ this tuning was performed by minimizing the difference between ionization potential and $-\epsilon_{\mathrm{H}}$, the eigenvalue of the KohnSham (KS) HOMO. Finally, for the study of the photochemical reactive decay path of solvated $5 \mathrm{BU}$ (as above, the solvent was modeled implicitly using PCM), a set of constrained and unconstrained geometry optimizations on the first excited state was performed using the M06 functional. For these calculations restricted KS LR-TDDFT was used. We verified, by comparing with energies obtained with unrestricted KS calculations at two points in the vicinity of bond formation, that a restricted description is justified.

\section{RESULTS AND DISCUSSION}

3.1. Validation of DFT/LR-TDDFT xc Functional. In this section, we investigate the performance of the different DFT functionals ranked according to the Perdew's ladder. For dark excitations, we compare directly the excited state energies of selected states with high level reference calculations performed at the CIS(D) and CC2 level of theory. These tests will be performed in the gas phase, a choice motivated by the fact that most high level calculations are not available in implicit solvent and results are not easily comparable with experimental results. For bright excitations we will validate our LR-TDDFT calculations based on experimental quantities (absorption spectra).

Validation in Gas Phase. Assessment of the Dark States. In ref 6 the first two electronic excited states of 5BU have been characterized in the Franck-Condon region using high level post-Hartree-Fock methods. It has been shown that the first excited state transition is dark and has mainly intrachromophore uracil $\mathrm{n} \pi^{*}$ character. The second excited state transition can be assigned to a uracil $\pi \pi^{*}$ transition. However, only the vertical excitation energy to the first excited state is 
well predicted by all selections of active spaces (the results are all between 4.93 and $5.02 \mathrm{eV}$ ). Instead, the vertical excitation energy to the second excited state depends strongly on the active space considered in the calculation. In particular, it was found that when multistate multideterminantal wave function expansions correlating only the valence $\pi$ and $\mathrm{n}$ orbitals are considered, the vertical excitation energy for this state is not reproduced accurately. The same effect was already noted in the case of the uracil moiety itself. ${ }^{37}$

The vertical excitation energies to the first three excited states obtained with high level calculations and with LRTDDFT with the different tested functionals are reported in Figure 2. We group the transitions into two main classes that are characterized by a different mixture of intrachromophore CT (CT within a single chromophore moiety) and interchromophore CT (between the two chromophores). In many cases, the transitions appear to have a mixed character and therefore it was necessary to introduce a third class for the mixed-character case. The assignement of the computed transitions to the different classes was based on the overlap between the hole and excess density produced upon excitation (orange and black densities in Figure 3). This classification is rather arbitrary, and it serves only to simplify the terms of the following discussion. An inspection of the character of the

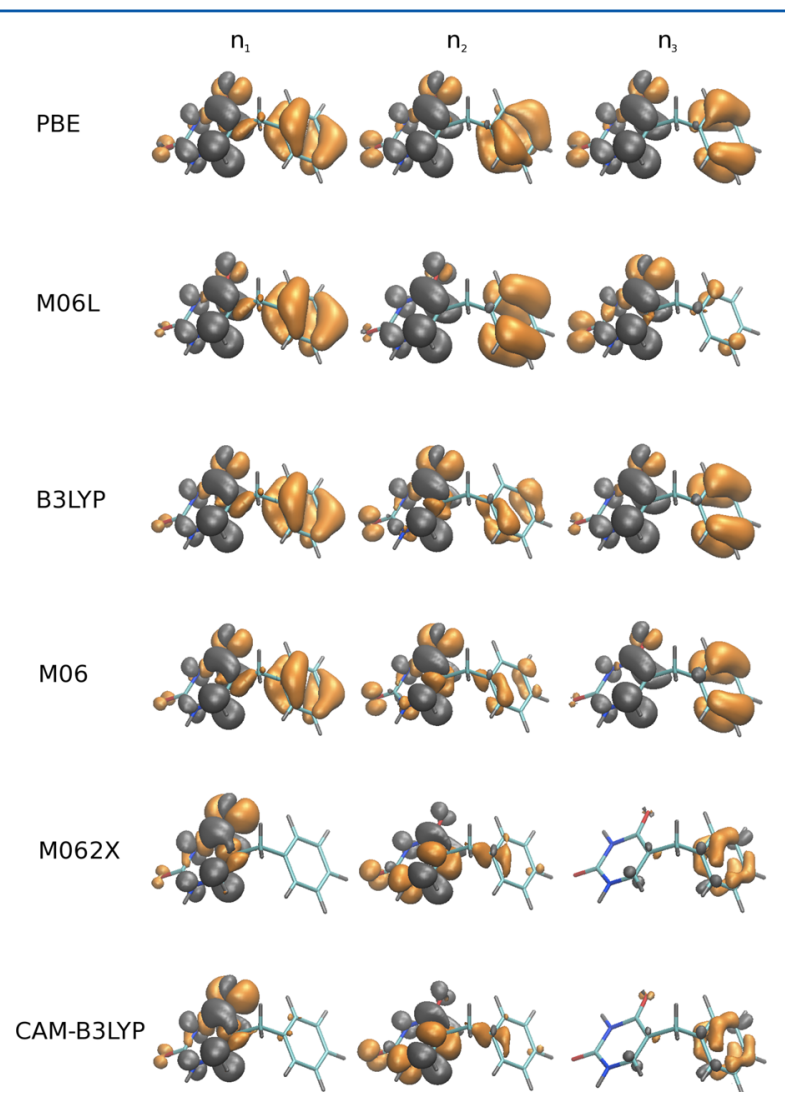

Figure 3. Isosurface plots corresponding to the first three excited states transition densities obtained in gas phase using the $\mathrm{xc}$ functionals indicated on the left. Densities are depicted with an isovalue of $\kappa=0.002$. The positive values are in gray (electron) and the negative values (hole) in orange. The charge transfer character of the transitions is apparent for all local functionals; transitions become localized within the individual chromophores (see the two bottom lines for $n_{1}$ and $n_{3}$ ) when the long-range correction is used. transitions in Figure 3 will help to go beyond this simplified picture.

The results for the different $\mathrm{xc}$ functionals (Figure 2) are organized following their order in the Perdew's ladder. All the predicted energies increase systematically from left to right, i.e., as we move up in Perdew's ladder. This is particularly true for the CT states (interchromophoric as well as mixed, intra- and interchromophore, transitions) shown in blue and black. As it can be observed, when the GGA approximation is used, the intrachromophore transitions (of mainly $\mathrm{n} \pi^{*}$ character in the intrauracil component) is underestimated by $\sim 0.6-0.8 \mathrm{eV}$ with respect to the higher level reference values (CC2). This indicates clearly that the most local approximations of the $\mathrm{xc}$ functional do not adequately describe the energy of this particular transition. Instead, starting from the meta-hybrid level of approximation (M06 functional), the energy of this type of transitions increases, suggesting improved predictivity for this class of $\mathrm{xc}$ functionals.

The interchromophore $\mathrm{CT}$ and the mixed (intra- and interchromophore) transitions involve also an important $\pi \pi^{*}$ component from both chromophores (black and blue solid lines in Figure 2, respectively). Both transitions have very similar CT character with hole on the benzene and excited electron on the uracil when PBE, M06L, B3LYP, and M06 functionals are used (see Figure 3). With M06-2X and CAMB3LYP instead, the character of the low-lying transitions (Figure 2, red lines) changes drastically, and they become almost pure intrachromophore transitions, typical of the two separated chromophore species (see Figure 3). In particular, the character of these transitions is now associated with the benzene dark $\pi \pi^{*}$ transition $(5.10 \mathrm{eV}$ in isolated benzene at the CASPT2 level of theory ${ }^{6}$ ) and the uracil dark $\mathrm{n} \pi^{*}$ and bright $\pi \pi^{*}$ transitions (4.54 and $5.0 \mathrm{eV}$ in isolated uracil at the CASPT2 level of theory $\left.{ }^{38}\right)$. The pure interchromophore CT transitions observed with the first set of functionals have now higher energies and are not shown. As mentioned in the Introduction, the energy of CT states is usually underestimated by the local and semilocal approximations, and the use of functionals like CAM-B3LYP and M06-2X is recommended in some cases. However, in ref 32 it was shown that the use of long-range corrections may not improve CT transitions when the overlaps between the occupied and virtual orbitals participating in the electronic transitions are larger than a given threshold. In these cases, the quality of the results may even worsen when this type of functionals are used. In the same work, a quantitative measure for the threshold, the so-called $\Lambda$ parameter, was introduced. This parameter measures the overlap of the orbitals participating in the electronic transition, and its calculation has been proposed as a diagnostic tool of the reliability of local and semilocal xc functionals in the evaluation of CT transition energies. In fact, it has been observed that (statistically) the CT excitation energies have significant errors if the value of the parameter falls below 0.5 for the GGA (PBE) and below 0.4 for hybrids (B3LYP). ${ }^{39,40}$ When the $\Lambda$ parameters associated with $\mathrm{CT}$ transitions are greater than 0.5 , on the other hand, the CT transitions are frequently well described by standard GGA functionals like PBE. The $\Lambda$ parameters obtained for the transitions of 5BU examined here lie in the intermediate overlap range between 0.45 and 0.65 . These values are above the critical threshold and seem to indicate that the degree of CT associated with these states is not due to the use semilocal xc functionals. 
As mentioned in the Computational Details section, to further investigate the impact of Hartree-Fock long-range corrections for our system, we also determined the optimal value of the separation parameter $\gamma$. Taking the GGA functional $\mathrm{BLYP},{ }^{35}$ the calculated optimal value is $\gamma=0.26 \mathrm{bohr}^{-1}$, below the standard value $\left(\sim 0.33 \mathrm{bohr}^{-1}\right)$ recommended for range separated functionals. ${ }^{36}$ This observation indicates that large fractions of exact exchange (as in M06-2X) or addition of exact exchange at long and short range (as in the asymptotically corrected hybrid CAM-B3LYP functional) may not necessarily improve the characterization of the intrachromophore transitions in $5 \mathrm{BU}$ in the gas phase.

In summary, while it is possible to observe a clear trend for the energies and the ordering of the states moving up the Perdew's ladder, it is still unclear if the range-separated functionals provide a better description of the absorption spectra of 5BU at its optimized geometry in gas phase or not. Additional calculations performed at CIS(D) and CC2 levels of theory are not sufficient to draw a final conclusion about the correct ordering of the states: CC2 predicts a first transition at $5.04 \mathrm{eV}$ with $\mathrm{n} \pi^{*}$ character, while the two $\pi \pi^{*}$ states lie slightly higher in energy (5.26 and $5.31 \mathrm{eV}$, with mainly intrachromophore and mixed character, respectively); CIS(D), on the contrary, has a similar behavior as the two range-separated hybrids, but all energies are strongly blue-shifted and lie above $6 \mathrm{eV}$.

The situation becomes less intricate as soon as we move away from the optimized ground state geometry along a reaction path that brings closer to the product, 5,6BU. To show this trend, we performed LR-TDDFT calculations with the structure characterized by a value of $R_{c}=-3 \AA$ along the reaction coordinate described in the following section (section 3.2). At this geometry, the orbital reorganization is such that all molecular orbitals not directly involved in the process are destabilized and shifted up in energy. The first and dominant transition, which is a mixture of intra- and intermolecular $\pi \pi^{*}$, is now well described by meta-hybrids (M06) and also rangeseparated hybrids (CAM-B3LYP) as shown in Figure 4 and Table 1. This was not the case at the ground state optimized geometry.

Validation in Solution: Assessment of the Bright States. The performance of the different functionals in calculations for solvated $5 \mathrm{BU}$ is assessed in the following by direct comparison with the experimental absorption spectrum. In this case, the relevant states are those associated with bright $\pi \pi^{*}$ transitions (the $\mathrm{n} \pi^{*}$ transitions have small oscillator strengths and

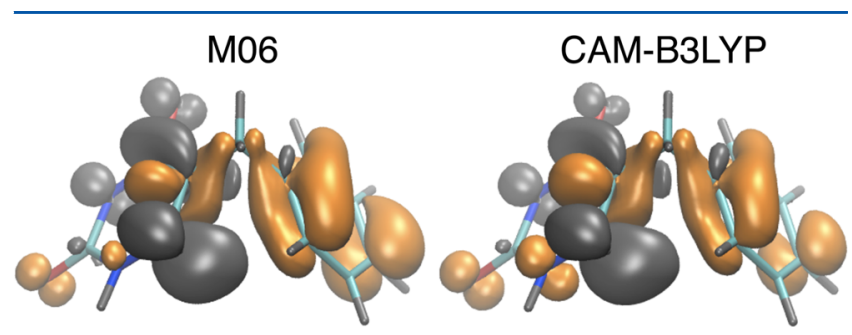

Figure 4. Isosurface plots of the transition densities in the gas phase using the M06 xc functional (on the left side) and the CAM-B3LYP xc functional (on the right side) computed for the structure characterized by a value of $R_{c}=-3 \AA$ along the reaction coordinate described in section 3.2 and Figure 7. The isovalue is set to $\kappa=0.002$. Positive density differences are in gray (electron) and negative (hole) in orange.
Table 1. Gas Phase Excitation Energies (in eV), State Characters, and Oscillation Strengths of 5BU Computed for the Geometry at $R_{\mathrm{c}}=-3 \AA$ (See Figure 7) Using the M06 and CAM-B3LYP Functionals

$\begin{array}{lccc}\text { functional } & \text { energy }(\mathrm{eV}) & \text { character } & \text { oscillator strength } \\ \text { M06 } & 3.44 & \text { CT } \pi \pi^{*} & 0.07 \\ \text { CAM-B3LYP } & 3.78 & \text { CT } \pi \pi^{*} & 0.01\end{array}$

therefore they cannot be observed experimentally). Calculations were performed using (i) the M06 functional which, among the local and semilocal tested functionals, provides the best agreement in the gas phase, (ii) the M06-2X and CAMB3LYP to investigate further the effect of using massive exact exchange at all distances (M06-2X) and range separated functionals with exact exchange at long-range (CAM-B3LYP), and (iii) the PBE functional (even though it is known to be less accurate, this functional was tested in view of future calculations; see section 3.2).

In Figure 5, we show the experimental absorption spectra (continuous black curves), together with the theoretical predictions obtained with different functionals. The tested functionals, except for PBE, correctly predict the bimodal shape of the spectrum (even if the spectra are blue-shifted in all cases). The error in the transition energies can be estimated by comparing the position of the lowest energy peak in the computed spectrum with the experimental one. The M06 functional (upper left panel) yields the most accurate results with an error of only $0.26 \mathrm{eV}$. This result confirms that transition energies are well described by this functional. With M06-2X and CAM-B3LYP (upper right panel and lower left panel, respectively) excitation energies are instead slightly overestimated for this system. Consistently with the gas phase calculations, the PBE functional (lower right panel) underestimates the transition energies. Moreover, the predicted spectrum loses the experimental bimodal shape. In Table 2, we report the energies for the first two excited states for different functionals.

In Figure 6, we show the isosurfaces of the transition densities corresponding to the two lowest lying electronic excitations. At variance with what observed in the gas phase, the intrauracil moiety transitions of $\mathrm{n} \pi^{*}$ and $\pi \pi^{*}$ characters are considerably mixed. The origin of this mixing can be attributed to solvent polarization effects on the $\pi \pi^{*}$ and $\mathrm{n} \pi^{*}$ transitions in uracil already reported in the literature. This solvatochromic effect was in fact observed in studies of uracil in solution performed using both LR-TDDFT ${ }^{41}$ and wave function based approaches. ${ }^{42}$ Moreover, again at variance with the gas phase, the lowest intrachromophore transitions located on the benzene moiety are found at energies higher than $6 \mathrm{eV}$ (results not shown). Finally, Figure 6 also shows how the CT contribution of these transitions decreases systematically along the sequence in Figure 6. In particular, with PBE the CT component is dominant for both excitations, while with M06 a strong CT character is observed only for the first excitation (in which the uracil $\pi \pi^{*}$ transition is also dominant). ${ }^{43}$ When the CAM-B3LYP and M06-2X functionals are considered, all CT contributions become negligible, and the transitions are mainly localized on the uracil, with only a slight perturbation induced by the aromatic $\pi$ electrons of the benzene. The $\Lambda$ parameters associated with the transitions (also reported in Figure 6) indicate that in all cases the orbital 

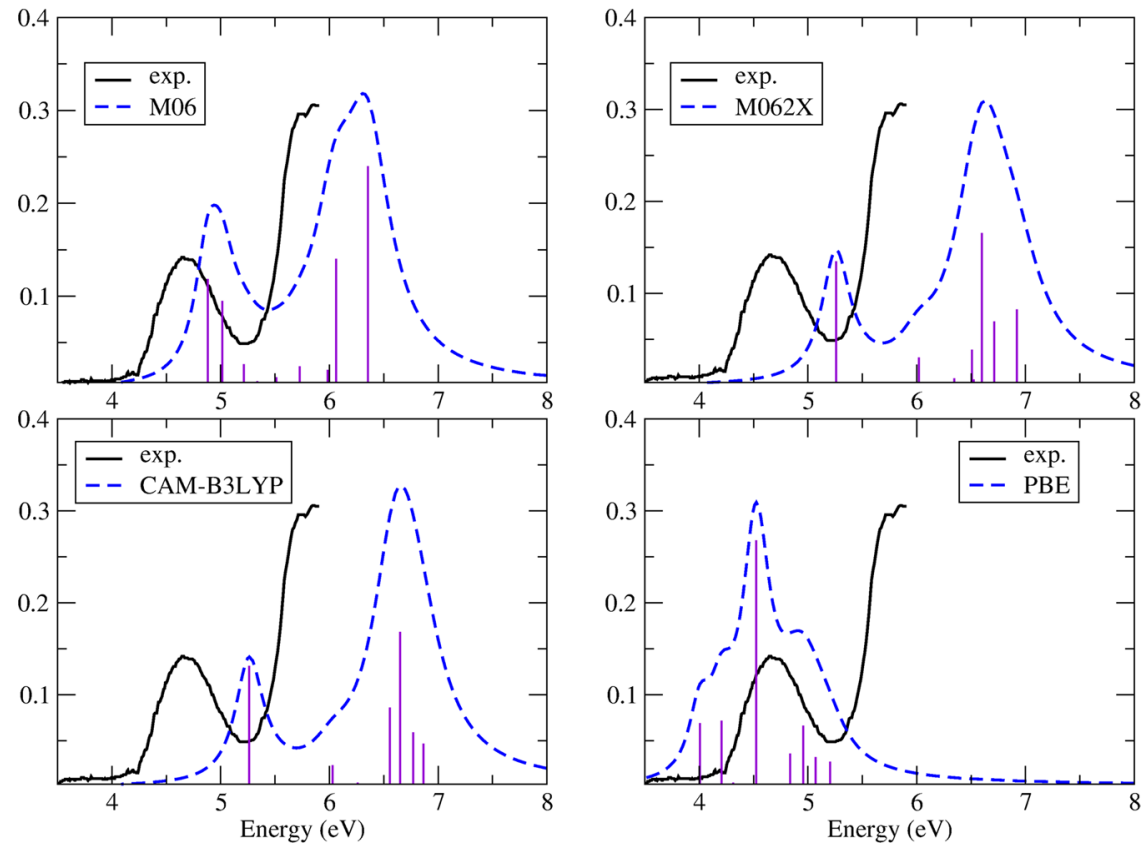

Figure 5. Absorption spectra in solution (blue dashed lines) obtained with the functional indicated in the inset. The curves are Gaussian convolutions of the computed spectral lines (vertical purple lines) weighted by their corresponding oscillator strengths. The excited state calculations are performed using the optimized structures corresponding to the specified functional. The solid black lines correspond to the experimental spectrum in $\mathrm{MeOH}$.

Table 2. Excitation Energies (in eV) for the First Two Singlet Excited States in the Solvated Phase for the Tested xc Functionals $^{a}$

\begin{tabular}{clllll} 
& \multicolumn{1}{c}{ PBE } & M06 & M06-2X & CAM-B3LYP & exp \\
\hline $\mathrm{S}_{0} \rightarrow \mathrm{S}_{1}$ & $4.01(0.02)$ & $4.88(0.12)$ & $5.22(0.01)$ & $5.26(0.26)$ & $5.33(0.00)$ \\
$\mathrm{S}_{0} \rightarrow \mathrm{S}_{2}$ & $4.21(0.02)$ & $5.01(0.10)$ & $5.26(0.27)$ & 5.26 & 4.68
\end{tabular}

${ }^{a}$ Oscillator strengths are reported in parentheses. The last line reports the position of the lowest energy peak obtained after Lorentian broadening of the TDDFT transitions (with full width at half-maximum of $0.25 \mathrm{eV}$ ).

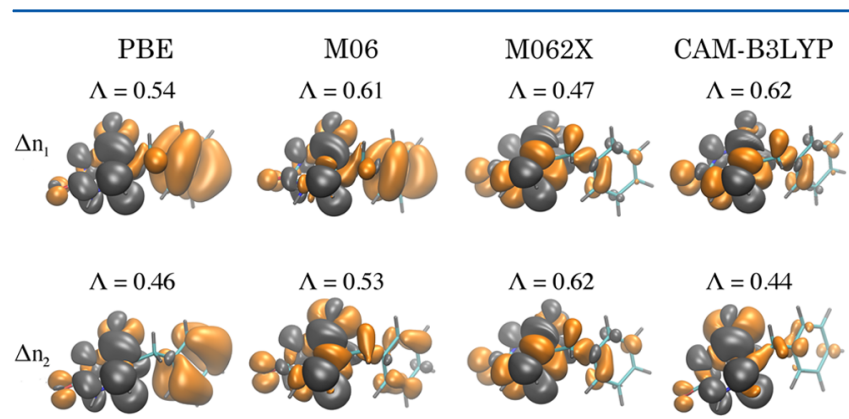

Figure 6. Isosurface plots of the transition densities for the first $\left(\Delta n_{1}\right.$, upper line) and second $\left(\Delta n_{2}\right.$, bottom line) excitation of $5 B \mathrm{BU}$ in implicit solvent. Each column reports $\Delta n_{1}$ and $\Delta n_{2}$ for the xc functional indicated at the top. The isovalue is set to $\kappa=0.0007$. We used a smaller value than in Figure 3 in order to better capture the decrease of the CT component when going from PBE to CAMB3LYP. Positive density differences are in gray (electron) and negative (hole) in orange.

overlap lies in the intermediate range in which the long-range corrections can worsen the quality of the results.

3.2. Description of the Reactive Decay Path. Based on the analysis reported so far, reliable LR-TDDFT results are obtained when the M06 functional is used. In particular, moving along the reaction path that connects the initial structure $(5 \mathrm{BU})$ to the photoreaction product $(5,6 \mathrm{BU})$ the differences between meta-hybrid functionals (M06) and rangeseparated hybrids (CAM-B3LYP) tend to vanish. In the case of $5 \mathrm{BU}$ in solution, we also found that the use of range separated hybrids worsens slightly rather than improving the quality of the absorption spectrum. We believe that due to the large value of the $\Lambda$ parameter, the M06 functional gives the most accurate and balanced description of the different excited states in 5BU (intra- and interchromophores).

In this section, we will therefore use the M06 energies to benchmark the performance of the PBE functional along the decay path identified in ref 2 and summarized below as a possible route to 5,6BU formation after photoexcitation. A general picture of the deactivation mechanisms of $5 \mathrm{BU}$ in solution has already been suggested in ref 2 based on minimum-energy path calculations (geometry relaxations in the electronic excited state) performed using the M06 functional. In particular, it has been shown that the relaxation of the molecule on the second excited state PES (whose main character is given by the uracil $n \pi^{*}$ state) is associated with the fluorescence emission of the molecule. Two possible decay paths were also identified on the PES corresponding to the bright $\pi \pi^{*}$ excited state in the Franck-Condon region. Along the first relaxation path, the deactivation mechanism implicates only the uracil moiety. Its structural changes upon relaxation involve a puckering motion of the excited pyrimidine DNA heterocycle. This motion is known to be linked to nonradiative 


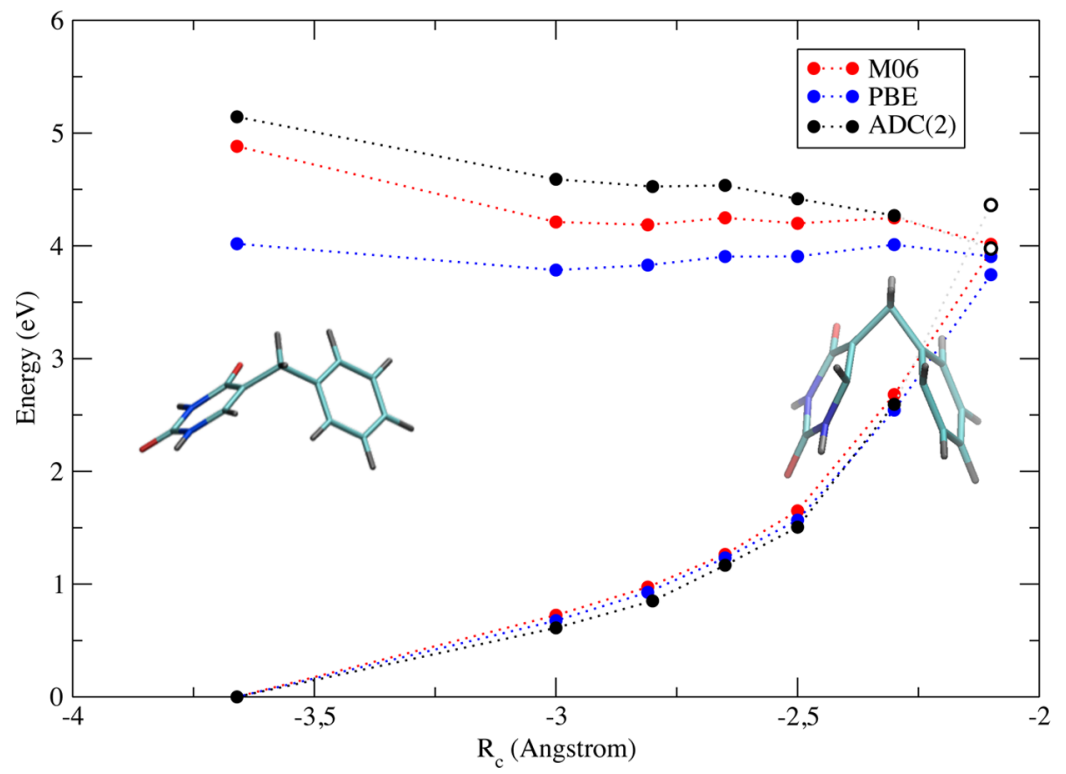

Figure 7. Energy scan of the excited zwitterionic PES using the M06 xc functional (in red). The reaction coordinate $\left(R_{c}\right)$ corresponds to the negative of the $\mathrm{C} 6-\mathrm{C} 10$ distance. The results obtained using the PBE xc functional are also reported (blue) together with points computed at $\mathrm{ADC}(2)$ level of theory (black). Open black circles indicate the point of instability of the $\operatorname{ADC}(2)$ calculation, which is characterized by a negative excitation energy. Insets show the structure of solvated $5 \mathrm{BU}$ at the ground state equilibrium geometry (left) and for a C6-C10 distance of $2.1 \AA$ (right).

decay paths via conical intersections. ${ }^{37,38,41,42,44}$ The other channel, instead, involves both chromophores and is driven by the electrostatic coupling induced by the interchromophore CT transition discussed in the previous section. Depending specifically on the perturbations induced by the presence of the phenyl moiety, this decay channel is characteristic of 5BU and has never been observed in other nucleobase derivatives.

Following the idea that this last decay path is the one responsible for the photoinduced cyclization of 5BU, we performed a scan of the $\pi \pi^{*}$ excited state PES with M06 along the reaction coordinate $R_{\mathcal{c}}$ defined as the negative of the C6C10 atomic distance (see Figure 1c for the atoms labeling). These are the two atoms forming the extra bond in the photoproduct 5,6BU (Figure 1d). The results obtained are reported in Figure 7 (red curve). The energy at $R_{\mathrm{c}}=-3.66 \AA$ is computed using an unconstrained geometry optimization on the ground state PES (and represents the equilibrium structure in the Franck-Condon region). Similarly, the energy at $-2.81 \AA$ corresponds to the optimized geometry on the CT PES, which is a local minimum on this surface. The energies at $R_{\mathrm{c}}=-3.0 \AA, R_{\mathrm{c}}=-2.5 \AA$, and $R_{\mathrm{c}}=-2.1 \AA$ are obtained via constrained optimizations in which all molecular degrees of freedom are allowed to change except for the ones associated with the distance $R_{c}$. The others energies have been extrapolated with single point calculations on the average nuclear coordinate between two optimized geometries. Figure 7 shows that there is at least one monotonically decreasing energetic path on this surface that brings the two atoms to a distance of $2.1 \AA$. The electronic configuration of the two carbon atoms (C6 and C10) changes dramatically along the path. In the reactant state (structure on the left in Figure 7) the carbons have a $\mathrm{sp}^{2}$ planar configuration. At the end of the path they assume a pyramidal $\mathrm{sp}^{3}$ hybridization allowing for covalent bond formation between them (structure on the right in Figure 7). Moreover, the small energy gap between excited and ground state PESs at $R_{\mathrm{c}} \simeq-2.1 \AA$ (less than $0.1 \mathrm{eV}$ ) opens the possibility of nonadiabatic transitions back on the ground state
PES. It can be expected that the excess kinetic energy accumulated along the path can drive the two carbon atoms at closer distance than what shown in Figure 7. In this case, if short enough distances are reached during the relaxation motion, the covalent bond formation between the two atoms becomes possible (also in the ground state). Another possible competing reaction path could, after the nonadiabatic transition to the ground state, drive the system back to the starting ground state equilibrium structure (following the ground state PES gradients pointing toward the starting equilibrium ground state configuration).

An accurate description of the bond formation mechanism requires the application of $a b$ initio molecular dynamics (MD) methods in which the description of nonadiabatic effects are included. This type of simulation can be efficiently performed using the LR-TDDFT based trajectory surface hopping approach to nonadiabatic dynamics. ${ }^{45-48}$

In view of the possibility to perform nonadiabatic dynamic calculations on this photoinduced cyclization process, we tested the performance of computationally less demanding functionals of the GGA family. In particular, in Figure 7 we report energy profiles along the reaction path coordinate $R_{c}$ using TDDFT with the functionals PBE (blue curve), M06 (red curve), and $\operatorname{ADC}(2)^{49}$ (black curve). All calculations are performed using the implicit solvent model COSMO as described in section 2 . We note that both TDDFT curves are in qualitative good agreement with the reference $\operatorname{ADC}(2)$ calculation. Even though PBE fails in the description of the PES in the Franck-Condon region, the two TDDFT PESs become parallel for values of $\left|R_{c}\right|$ shorter than $3 \AA$. It is important to mention that $\operatorname{ADC}(2)$ produces instabilities close to the crossing points with the ground state where we obtained a negative value for the excitation energy (open black circles in Figure 7). This pathology of $\mathrm{ADC}(2)$ is already well documented in the literature. ${ }^{50}$ Further tests using nonadiabatic dynamics approaches are required to determine which functional (e.g., M06 or PBE) is better suited (i.e., reliable and computationally 
affordable) for the simulation of this photocyclization process and to assess the effect of the small barrier on the M06 curve.

\section{CONCLUSIONS}

We showed that when the M06 xc energy functional is used, LR-TDDFT provides a good description of the photophysical and photochemical properties of 5BU, becoming a valid alternative to more sophisticated wave function based calculations, which are computationally too expensive for dynamics.

In the gas phase, while it is possible to detect a trend for the energies and characters of the states as one moves up the Perdew's ladder of functionals, we could not identify a clear sign of convergence; the effect of adding more exact-exchange at short and long separations is hard to predict and can be very different for states having different amount of CT character, as measured by the parameter $\Lambda$. However, we could find points in the configuration space for which the differences among the functionals became less important, therefore making the selection of the functional more straightforward and less arbitrary.

Adopting the implicit solvent model, we observed a good agreement with experimental data in the evaluation of the lowest bright $\pi \pi^{*}$ states transition energies in solution (within an error of $0.26 \mathrm{eV}$ ). Tests performed using different xc energy functionals show that with local and semilocal functionals transition energies are severely underestimated due to the CT character associated with the interchromophore transitions. We also showed that in 5BU the use of M06-2X or CAM-B3LYP does not necessarily improve the results. On the contrary, the transition energies are overestimated and the CT presence in the character of the states might be spuriously underpredicted.

Concerning the reactive path, the presence of the strong CT character in the lowest bright $\pi \pi^{*}$ electronic transition predicted by the M06 functional may play a critical role in explaining the photoreactivity of the 5BU: the zwitterionic character of $5 \mathrm{BU}$ in this excited state induces a relaxation motion that can lead to the covalent bond formation observed in the photoproduct. The performance of the PBE functional for the calculation of the PESs along this putative reaction path was investigated in view of future LR-TDDFT-based nonadiabatic MD calculations. These simulations will allow the study of the bond formation mechanism at the base of the cyclization photoreaction that from $5 \mathrm{BU}$ leads to 5,6BU. We found that even though the GGA approximation of the $\mathrm{xc}$ functional cannot describe the photophysical properties of the molecule in the neighborhood of the Franck-Condon region, a qualitative agreement with the M06 results is obtained in regions of the configurational space far from the FranckCondon region but relevant for the study of the decay path.

\section{AUTHOR INFORMATION}

\section{Corresponding Author}

*E-mail: ita@zurich.ibm.com (I.T.).

\section{ORCID 1}

I. Tavernelli: 0000-0001-5690-1981

\section{Notes}

The authors declare no competing financial interest.

\section{ACKNOWLEDGMENTS}

M.M. and S.B. acknowledge financial support from the Italian Institute of Technology (Grant IIT-SEED Project N 259
SIMBEDD). I.T. acknowledges the COST action XLIC, the Swiss National Science Foundation (SNF) through Grant 200021-146396, and the Swiss NCCR national project MARVEL for funding. We also thank Giovanni Ciccotti for interesting discussions.

\section{REFERENCES}

(1) Sun, G.; Fecko, C.; Nicewonger, R.; Webb, W.; Begley, T. DNAProtein Cross-Linking: Model Systems for Pyrimidine-Aromatic Amino Acid Cross-Linking. Org. Lett. 2006, 8, 681-683.

(2) Micciarelli, M.; Valadan, M.; Della Ventura, B.; Di Fabio, G.; De Napoli, L.; Bonella, S.; Rothlisberger, U.; Tavernelli, I.; Altucci, C.; Velotta, R. Photophysics and Photochemistry of a DNA/Protein Cross-Linking Model: A Synergistic Approach Combining Experiments and Theory. J. Phys. Chem. B 2014, 118, 4983-4992.

(3) Dreuw, A.; Head-Gordon, M. Failure of Time-Dependent Density Functional Theory for Long-Range Charge-Transfer Excited States: The Zincbacteriochlorin-Bacteriochlorin and Bacteriochlorophyll-Spheroidene Complexes. J. Am. Chem. Soc. 2004, 126, 40074016.

(4) Magyar, J. R; Tretiak, S. Dependence of Spurious ChargeTransfer Excited States on Orbital Exchange in TDDFT: Large Molecules and Clusters. J. Chem. Theory Comput. 2007, 3, 976-987.

(5) Gritsenko, O.; Baerends, E. J. Asymptotic Correction of the Exchange-Correlation Kernel of Time-Dependent Density Functional Theory for Long-Range Charge-Transfer Excitations. J. Chem. Phys. 2004, 121, 655-660.

(6) Micciarelli, M.; Altucci, C.; Della Ventura, B.; Velotta, R.; Tos, V.; Gonzalez Perez, A.; Perez, M.; de Lera, A.; Bende, A. Low-Lying Excited-States of 5-Benzyluracil. Phys. Chem. Chem. Phys. 2013, 15, $7161-7173$

(7) Fabiano, E.; Lan, Z.; Lu, Y.; Thiel, W. In Conical Intersections: Theory, Computation and Experiment; Domcke, W., Yarkony, D., Koppel, H., Eds.; World Scientific Publishing Company: 2011; pp 463-496.

(8) Silva-Junior, M.; Thiel, W. Benchmark of Electronically Excited States for Semiempirical Methods: MNDO, AM1, PM3, OM1, OM2, OM3, INDO/S, and INDO/S2. J. Chem. Theory Comput. 2010, 6, $1546-1564$.

(9) Perdew, J. P.; Schmidt, K. Density Functional Theory and Its Application to Materials; AIP: Melville, NY; pp 1-20.

(10) Perdew, J.; Burke, K.; Ernzerhof, M. Generalized Gradient Approximation Made Simple. Phys. Rev. Lett. 1996, 77, 3865-3868.

(11) Tavernelli, I. Electronic Density Response of Liquid Water using Time-Dependent Density Functional Theory. Phys. Rev. B: Condens. Matter Mater. Phys. 2006, 73, 094204.

(12) Tapavicza, E.; Tavernelli, I.; Rothlisberger, U. Trajectory Surface Hopping within Linear Response Time-Dependent Density-Functional Theory. Phys. Rev. Lett. 2007, 98, 023001.

(13) Curchod, B. F. E.; Rothlisberger, U.; Tavernelli, I. TrajectoryBased Nonadiabatic Dynamics with Time-Dependent Density Functional Theory. ChemPhysChem 2013, 14, 1314-1340.

(14) Tavernelli, I. Nonadiabatic Molecular Dynamics Simulations: Synergies Between Theory and Experiments. Acc. Chem. Res. 2015, 48, 792.

(15) Casida, M. E. Low-Lying Potential Energy Surfaces; American Chemical Society, 1st ed.; Chapter 9, pp 199-220.

(16) Zhao, Y.; Truhlar, D. G. A New Local Density Functional for Main-Group Thermochemistry, Transition Metal Bonding, Thermochemical Kinetics, and Noncovalent Interactions. J. Chem. Phys. 2006, $125,194101$.

(17) Stephens, P.; Devlin, F.; Chabalowski, C.; Frisch, M. Ab-Initio Calculation of Vibrational Absorption and Circular-Dichroism Spectra using Density-Functional Force-Fields. J. Phys. Chem. 1994, 98, $11623-11627$.

(18) Zhao, Y.; Truhlar, D. The M06 Suite of Density Functionals for Main Group Thermochemistry, Thermochemical Kinetics, Noncovalent Interactions, Excited States, and Transition: Two New 
Functionals and Systematic Testing of Four M06-Class Functionals and 12 other Functionals. Theor. Chem. Acc. 2008, 120, 215-41.

(19) Yanai, T.; Tew, D.; Handy, N. A New Hybrid ExchangeCorrelation Functional Using the Coulomb-Attenuating Method (CAM-B3LYP). Chem. Phys. Lett. 2004, 393, 51-57.

(20) Li, R.; Zheng, J.; Truhlar, D. G. Density Functional Approximations for Charge Transfer Excitations with Intermediate Spatial Overlap. Phys. Chem. Chem. Phys. 2010, 12, 12697-12701.

(21) Kendall, R.; Dunning, T. H.; Harrison, R. Electron Affinities of the First-Row Atoms Revisited. Systematic Basis Sets and Wave Functions. J. Chem. Phys. 1992, 96, 6796-6806.

(22) Ahlrichs, R.; Bar, M.; Haser, M.; Horn, H.; Kolmel, C. Electronic-Structure Calculations on Workstation Computers - the Program System Turbomole. Chem. Phys. Lett. 1989, 162, 165-169.

(23) Hattig, C.; Weigend, F. CC2 Excitation Energy Calculations on Large Molecules Using the Resolution of the Identity Approximation. J. Chem. Phys. 2000, 113, 5154.

(24) Schirmer, J. Beyond the Random-Phase Approximation - a New Approximation Scheme for the Polarization Propagator. Phys. Rev. A: At., Mol., Opt. Phys. 1982, 26, 2395-2416.

(25) Hattig, C. Structure Optimizations for Excited States with Correlated Second-Order Methods: CC2 and $\operatorname{ADC}(2) . A d v$. Quantum Chem. 2005, 50, 37-60.

(26) Tomasi, J.; Mennucci, B.; Cammi, R. Energetics, Stability, and Prediction of Transmembrane Helices. Chem. Rev. 2005, 105, 29993093.

(27) Gaussian09, Revision D.01; Gaussian Inc.: Wallingford, CT, 2009

(28) Cascella, M.; Cuendet, M. A.; Tavernelli, I.; Rothlisberger, U. Optical Spectra of $\mathrm{Cu}(\mathrm{II})$-Azurin by Hybrid TDDFT-Molecular Dynamics Simulations. J. Phys. Chem. B 2007, 111, 10248.

(29) Pedone, A.; Bloino, J.; Monti, S.; Prampolini, G.; Barone, V. Absorption and Emission UV-Vis Spectra of the TRITC Fluorophore Molecule in Solution: a Quantum Mechanical Study. Phys. Chem. Chem. Phys. 2010, 12, 1000-1006.

(30) Timrov, I.; Micciarelli, M.; Rosa, M.; Calzolari, A.; Baroni, S. Multimodel Approach to the Optical Properties of Molecular Dyes in Solution. J. Chem. Theory Comput. 2016, 12, 4423-4429.

(31) Crespo-Otero, R; Barbatti, M. Spectrum Simulation and Decomposition with Nuclear Ensemble: Formal Derivation and Application to Benzene, Furan and 2-Phenylfuran. Theor. Chem. Acc. 2012, 131, 1237.

(32) Peach, M. J. G.; Benfield, P.; Helgaker, T.; Tozer, D. J. Excitation Energies in Density Functional Theory: An Evaluation and a Diagnostic Test. J. Chem. Phys. 2008, 128, 044118.

(33) Gordon, M.; Schmidt, M. W. In Theory and Applications of Computational Chemistry: the First Forty Years; Dykstra, C. E., Frenking, G., Kim, K. S., Scuseria, G., Eds.; Elsevier: Amsterdam, 2005; pp 1167-1189.

(34) Iikura, H.; Tsuneda, T.; Yanai, T.; Hirao, K. A Long-Range Correction Scheme for Generalized-Gradient-Approximation Exchange Functionals. J. Chem. Phys. 2001, 115, 3540-3544.

(35) Becke, A. D. Density-Functional Exchange-Energy Approximation with Correct Asymptotic Behavior. Phys. Rev. A: At., Mol., Opt. Phys. 1988, 38, 3098-3100.

(36) Stein, T.; Kronik, L.; Baer, R. Reliable Prediction of Charge Transfer Excitations in Molecular Complexes Using Time-Dependent Density Functional Theory. J. Am. Chem. Soc. 2009, 131, 2818-2820.

(37) Matsika, S. Radiationless Decay of Excited States of Uracil through Conical Intersections. J. Phys. Chem. A 2004, 108, 7584-7590.

(38) Lorentzon, J.; Fulscher, M.; Roos, B. Theoretical-Study of the Electronic-Spectra of Uracil and Thymine. J. Am. Chem. Soc. 1995, 117, 9265-9273.

(39) Peach, J. G.; Benfield, P.; Helgaker, T.; Tozer, D. J. Excitation Energies in Density Functional Theory: An Evaluation and a Diagnostic Test. J. Chem. Phys. 2008, 128, 044118.

(40) Li, R.; Zheng, J.; Truhlar, D. G. Density Functional Approximations for Charge Transfer Excitations with Intermediate Spatial Overlap. Phys. Chem. Chem. Phys. 2010, 12, 12697.
(41) Improta, R.; Barone, V. Absorption and Fluorescence Spectra of Uracil in the Gas Phase and in Aqueous Solution: A TD-DFT Quantum Mechanical Study. J. Am. Chem. Soc. 2004, 126, 1432014321.

(42) DeFusco, A.; Ivanic, J.; Schmidt, M. W.; Gordon, M. S. SolventInduced Shifts in Electronic Spectra of Uracil. J. Phys. Chem. A 2011, $115,4574-4582$.

(43) This case illustrates the relative value of the $\Lambda$ parameter as an indicator of the CT character of a transition. In fact, one can have low values of $\Lambda$ also for intrachromophore transitions when there is minimal overlap between the electron and hole densities.

(44) Peach, M. J. G.; Cohen, A. J.; Tozer, D. J. Influence of Coulomb-Attenuation on Exchange-Correlation Functional Quality. Phys. Chem. Chem. Phys. 2006, 8, 4543-4549.

(45) Tavernelli, I.; Curchod, B.; Rothlisberger, U. Nonadiabatic Molecular Dynamics with Dolvent Effects: A LR-TDDFT QM/MM Study of Ruthenium (II) tris (Bipyridine) in Water. Chem. Phys. 2011, 391, 101-109.

(46) Capano, G.; Chergui, M.; Rothlisberger, U.; Tavernelli, I.; Penfold, T. J. A Quantum Dynamics Study of the Ultrafast Relaxation in a Prototypical Cu (I)-Phenanthroline. J. Phys. Chem. A 2014, 118, 9861-9869.

(47) Capano, G.; Rothlisberger, U.; Tavernelli, I.; Penfold, T. J. Theoretical Rationalization of the Emission Properties of Prototypical $\mathrm{Cu}$ (I)-Phenanthroline Complexes. J. Phys. Chem. A 2015, 119, 70267037.

(48) Franco de Carvalho, F.; Tavernelli, I. Nonadiabatic Dynamics with Intersystem Crossings: A Time-Dependent Density Functional Theory Implementation. J. Chem. Phys. 2015, 143, 224105.

(49) The choice of $A D C(2)$ rather than CC2 is motivated by the fact that $\mathrm{CC} 2$ calculations with the COSMO model (implicit solvent) is not fully implemented in latest distribution of the Turbomole code (missing response terms).

(50) Tuna, D.; Lefrancois, D.; Polanski, L.; Gozem, S.; Schapiro, I.; Andruniow, T.; Dreuw, A.; Olivucci, M. Assessment of Approximate Coupled-Cluster and Algebraic- Diagrammatic-Construction Methods for Ground- and Excited-State Reaction Paths and the ConicalIntersection Seam of a Retinal- Chromophore Model. J. Chem. Theory Comput. 2015, 11, 5758-5781. 